\title{
Primate Motor Cortex and Free Arm Movements to Visual Targets in Three-Dimensional Space. III. Positional Gradients and Population Coding of Movement Direction from Various Movement Origins
}

\author{
Ronald E. Kettner, ${ }^{a}$ Andrew B. Schwartz, ${ }^{b}$ and Apostolos P. Georgopoulos \\ The Philip Bard Laboratories of Neurophysiology, Department of Neuroscience, The Johns Hopkins University, School of \\ Medicine, Baltimore, Maryland 21205
}

In one experiment, we studied the relations between the frequency of discharge of $\mathbf{2 7 4}$ single cells in the arm area of the motor cortex of the monkey and the actively maintained position of the hand in space. We found that the frequency of discharge of $63.9 \%$ of the cells studied was a multilinear function of the position of the hand in space according to the following equation (multiple linear regression):

$$
d=f+f_{x} s_{x}+t_{y} s_{y}+f_{z} s_{z}
$$

where $d$ is the discharge rate of a single cell, $f, f_{x j}, f_{y}, f_{z}$ are regression coefficients, and $s_{x}, s_{y}, s_{z}$ are the coordinates of the position of the hand. The equation above defines a positional gradient which implies that the frequency of cell discharge will increase at a maximum rate when the position of the hand changes along a certain direction; we call this direction the orientation of the positional gradient, and the rate of change in discharge rate along this orientation, the magnitude of the gradient. The orientations of the positional gradients were distributed throughout three-dimensional (3D) space and their magnitudes differed among different cells.

In a different experiment, we studied the changes in activity of 289 cells in the arm area of the motor cortex when the monkeys made equal-amplitude movements that started from different points in space, were in the same direction, and traveled along parallel trajectories in 3-D space. Four pairs of such movement directions (i.e., a total of 8 movement directions) were studied for every cell, and the changes in cell activity associated with movements within each pair were compared. We found that these changes in cell activity did not differ statistically for $68.4 \%$ of the movement pairs

\footnotetext{
Received Aug. 3, 1987; revised Dec. 10, 1987; accepted Dec. 10, 1987

This work was supported by United States Public Health Service Grants NS 17413 and NS07226, which we gratefully acknowledge. We thank Mr. Joseph T. Lurito for help during some of the experiments. The color plot was produced using the Interactive Graphics Facility of the Department of Biophysics, The Johns Hopkins University, School of Medicine. This facility was established and maintained by NIH and NSF grants and by a gift from the Richard-King Mellon Foundation. R.E.K. was a research associate, 1985-1987. A.B.S. was a postdoctoral fellow, 1984-1987.

Correspondence should be addressed to Apostolos P. Georgopoulos, Bard Laboratories of Neurophysiology, Department of Neuroscience, The Johns Hopkins University, School of Medicine, 725 North Wolfe Street, Baltimore, MD 21205.

"Present address: Department of Psychology, Psychology Building, Indiana University, Bloomington, IN 47405.

"Present address: Division of Neurobiology, St. Joseph's Hospital and Medical Center, Barrow Neurological Institute, 350 West Thomas Road, Phoenix, AZ 85103.

Copyright (C) 1988 Society for Neuroscience $0270-6474 / 88 / 082938-10 \$ 02.00 / 0$
}

studied but did differ for the remaining $31.6 \%$. The data from the whole population of cells studied in this experiment were analyzed using the population vector analysis described in the preceding paper (Georgopoulos et al., 1988). Thus, 8 population vectors were calculated, 1 for each of the 8 movement directions studied. We found that the direction of the population vector was close to the direction of the corresponding movement. These results indicate that the population vector provides unique information concerning the direction of the movement even when the point of origin of the movement varies in 3-D space.

We described in the preceding 2 papers the relations between single-cell activity and the direction of movement (Schwartz et al., 1988), and a scheme by which the direction of movement in space could be uniquely encoded by a neuronal population (Georgopoulos et al., 1988). In those experiments the origin of the movement was the same; in the present studies we extended our observations to movements whose point of origin varied in the workspace. We found that the tonic discharge of many cells varied in an orderly fashion with the position at which the hand was actively maintained in space. Moreover, the changes in cell activity were similar in many cases for movements made in the same direction but in different parts of space, but differed for other cells; however, the population vector constructed from all the cells studied still pointed in the direction of movement even when the point of origin of the movement varied in space. Some preliminary results were presented previously (Kettner et al., 1986).

\section{Materials and Methods}

Animals. Two male rhesus monkeys were used. These were the same animals used in Schwartz et al. (1988).

Behavioral apparatus and tasks. The behavioral apparatus and training techniques used were described in the first paper of this series (Schwartz et al., 1988). Briefly, 9 lightable target buttons were placed in front of the animals as follows. The center button was at shoulder level in the midsagittal plane $15 \mathrm{~cm}$ away from the animal. The remaining 8 buttons were placed at equal distances $(12.5 \mathrm{~cm}$ ) from the central one, at the corners of an imaginary parallelepiped rotated in the horizontal plane $18^{\circ}$ towards the performing arm. The animals moved from onc light to another in a rcaction timc task. The sequence of events in a typical trial was as follows. The first light came on, and the monkey pushed it for a variable period of time of at least $0.5 \mathrm{sec}$, after which that light went off while a second light came on. The animal then moved to the second light, pushed it for at least $0.5 \mathrm{sec}$, and received a liquid reward. Figure 1 illustrates the tasks used. Figure $1 A$ shows the 9 positions of the target buttons at which the animal held its hand; and Figure $1, B, C$, illustrates the "center $\rightarrow$ out" and "parallel" tasks, 
respectively. The movements in the parallel task were of equal amplitude $(15.2 \mathrm{~cm})$. The tasks were performed separately in time, and each movement was repeated 5-8 times in a randomized block design.

Surgical, electrophysiological, and data collection techniques were as previously described (Schwartz et al., 1988).

\section{Data analysis}

Analysis epochs. The following epochs were distinguished for every trial. The control time (CT) was from the time that the first lighted button was pushed by the animal until the second light came on; the reaction time (RT) was from the time of onset of the second light until the hand left the first light; the movement time (MT) followed until the hand pushed the second light; and finally, during the ensuing target hold time (THT) the monkey held the hand at the second light until the reward was given. The combined RT and MT epochs were called the total experimental time (TET).

Positional gradient function. We calculated the positional gradient function from the center $\rightarrow$ out task using the frequency of discharge during the last $0.5 \mathrm{sec}$ of the THT (i.e., during holding at 8 peripheral positions) and the CT (i.e., during holding at center position). These "static" discharge rates were stable across repetitions for both the THTs and the CT.

First, an analysis of variance (ANOVA) was performed on the 9 statichold discharge rates. A statistically significant $(p<0.05) F$ test distinguished cells with a static positional effect. Then, a multiple linearregression analysis was carried out for the latter cells as follows. Let $s_{t}$, $s_{1}, s_{z}$ be the coordinates of a static-hold position with respect to the center of the workspace (i.e., the center light), and $d$ be the "static" discharge rate, as described above. The following regression equation was used:

$$
d(\mathbf{S})-f+f_{x} s_{x}+f_{y} s_{y}+f_{z} s_{z},
$$

where $d(\mathbf{S})$ is the discharge rate at position $\mathbf{S}=\left(s_{x}, s_{y}, s_{z}\right)$ and $f, f_{x}, f_{y}, f_{z}$ are regression coefficients. Equation 1 is similar in form, although not in content, to Equation 1 in Schwartz et al. (1988). A statistically significant $(p<0.05) F$ test distinguished cells with a positional gradient that was linear with respect to $x, y, z$ position. An equivalent form of Equation 1 is the following, which we call the positional gradient function:

$$
d(\mathbf{S})=f+h|\mathbf{S}| \cos \Theta_{\mathrm{GS}}
$$

where

$$
\begin{aligned}
h & =\left(f_{x}^{2}+f_{y}^{2}+f_{z}^{2}\right)^{1 / 2} \\
|\mathbf{S}| & =\left(s_{x}^{2}+s_{y}^{2}+s_{z}^{2}\right)^{1 / 2} \\
\mathbf{G} & =\left(g_{v}, g_{y}, g_{z}\right) \\
& =\left(f_{x} / h, f_{y} / h, f_{z} / h\right),
\end{aligned}
$$

and $\Theta_{\text {;;s }}$ is the angle between the vectors $\mathbf{G}$ and $\mathbf{S}$ (see Schwartz et al., 1988 , for more details on the derivation of this function). In Equation $2, f$ equals the discharge rate at the center button, and $h$ equals the rate of change in discharge rate with distance from the center button in the direction of $\mathbf{G} . \mathbf{G}$ is the direction of maximum change in the gradient and is therefore called the gradient orientation.

The positional gradient function (Eq. 2) is very similar to the directional tuning function (Eq. 4 in Schwartz et al., 1988) except for the added multiplicative factor $|\mathbf{S}|$, the distance from the origin. This added factor is not needed to specify movement direction but is required to uniquely specify position in space.

Single cell activity in the "parallel" task. In this task parallel movements were made from different points in space but in the same direction (Fig. 1C). Since the ongoing discharge rate differed for many cells while holding the arm at a certain position (see Results), we analyzed the changes in activity from the static discharge rate observed during the control time. An ANOVA was performed on these changes in discharge rate. It can be seen in Figure $1 C$ that 4 pairs of parallel movements in the same direction were made in this task. The average changes in cell activity associated with these paired movements were compared simultaneously using the "least significance difference" (LSD) method (Snedecor and Cochran, 1980). A nominally higher level of significance $(p<0.01)$ was used for statistical testing to compensate for the fact that 4 independent simultaneous comparisons were made. This probability
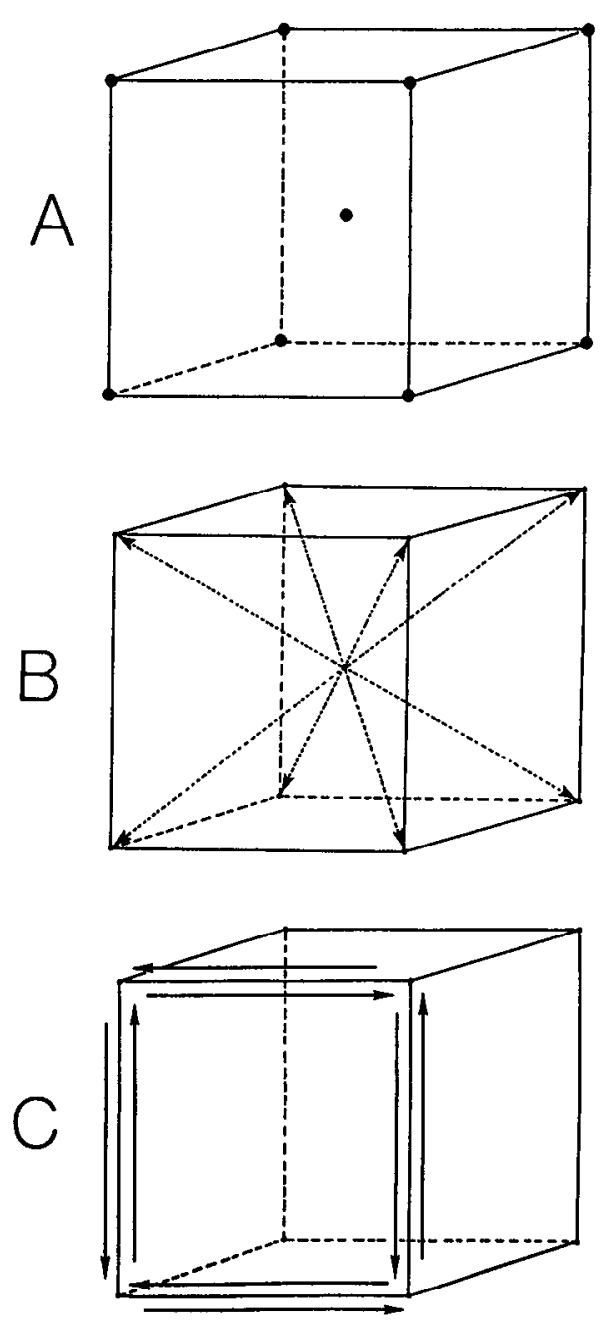

Figure 1. Layout of the workspace is shown for the left performing arm. A, Static hold positions; the dot inside the workspace was at the center of the workspace $(15 \mathrm{~cm}$ in front of the animal, in the midsagittal plane at shoulder level). $B$, "Center $\rightarrow$ out" task (movement amplitude $=12.5 \mathrm{~cm}$ ). $C$, "Parallel" task (movement amplitude $=15.2 \mathrm{~cm}$ ).

level was arrived at as follows (Bonferroni inequality; see Ingelfinger et al., 1983, p. 170). Assume that the level of significance chosen is $\alpha$. When $k$ simultaneous comparisons are being made, and, therefore, $k$ separate $p$ values are computed, there is a probability of up to $\alpha k$ that at least one $p$ value will be less than $\alpha$ even when the means compared do not differ. Under these conditions, a significance level of $\alpha / k$ is chosen as the adjusted criterion for "statistical significance." In the present case, the significance level desired is $\alpha=0.05$. Since 4 independent, simultaneous comparisons are being made, there is a probability of up to $4 \times 0.05=0.20$ that at least one $p$ value will be less than 0.05 even when the means compared do not actually differ. Therefore, the adjusted criterion for "statistical significance" was chosen to be $0.05 / 4=0.013$ $\approx 0.01$. It is obvious from the discussion above that although this level of significance testing is nominally lower than 0.05 , the actual testing is close to the 0.05 level.

Neuronal population analysis for movements of different origins. The purpose of this analysis was to compute the neuronal population vector for movements in the same direction from different origins and to compare the degree of congruence of the population vector with the direction of movement under those conditions. The calculations were based on 246 directionally tuned cells that were studied in both the center $\rightarrow$ out and the parallel tasks. The preferred direction of each of these cells was calculated using the changes in cell activity from the rate during the corresponding control time; all 16 available movement directions were used in the calculations. The population vector was computed as described in Georgopoulos et al. (1988) using the same weighting function as in that paper (weighting function 8 of Appendix 2 in 


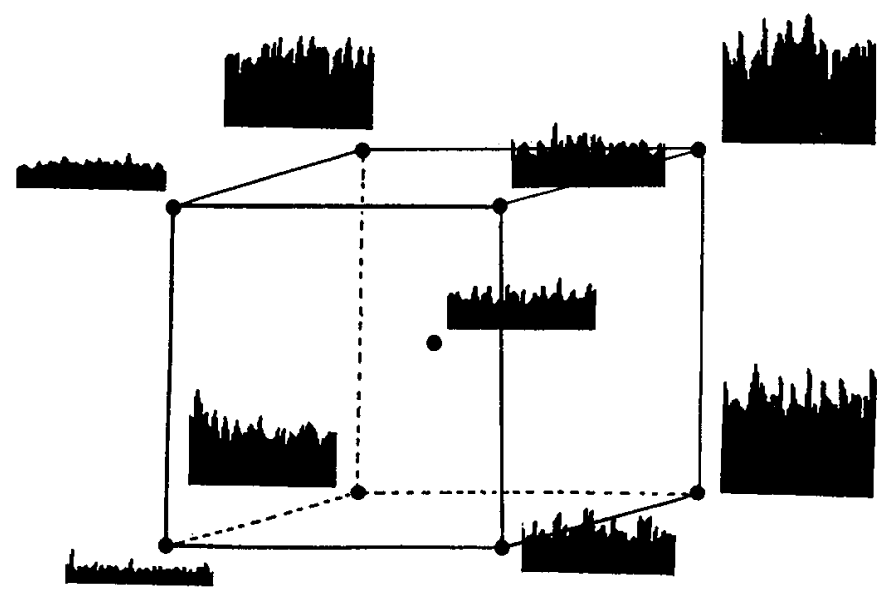

Figure 2. EMG activity of acromiodeltoid muscle during holding of thc hand at positions indicated. Each histogram is an average of 8 trials and is $0.5 \mathrm{sec}$ long.

Georgopoulos et al., 1988). Finally, the time course of the population vector was calculated every $20 \mathrm{msec}$ as described in Georgopoulos et al. (1988).

Electromyographic (EMG) analysis. The EMG activity of 5 muscles (acromiodeltoid, spinal deltoid, pectoralis major, biceps, and triceps) was recorded as described in Schwartz et al. (1988). The average voltage during the last $0.5 \mathrm{sec}$ of the THT and, for the center, CT epochs was used as a measure of EMG activity. The EMG signal was recorded from a limited volume of the muscle in which the multistranded EMG wires were inserted; no extensive sampling of other parts of a muscle was carried out. Therefore, the EMG data were regarded as qualitative observations and were not subjected to rigorous quantitative analysis.

\section{Results}

The data described below were obtained during the same microelectrode penetrations into the motor cortex of 2 monkeys as described by Schwartz et al. (1988) and depicted in Figure 6 of that paper.

\section{Positional effects}

EMG studies. The EMG activity varied among the positions tested. An example is shown in Figure 2 for the acromiodeltoid muscle. Conversely, holding at a certain position was associated with different levels of activity in several muscles (data not shown).

\section{Neuronal recording studies}

Positional gradient function. The frequency of discharge of 222/ $274(81.0 \%)$ cells studied differed significantly among different positions (ANOVA, $F$ test, $p<0.05$ ). The positional gradient function (Eq. 2) characterized the discharge of 175 of these 222 cells (multiple regression, $F$ test, $p<0.05$ ). An example is shown in Figure 3. The multiple regression equation for this cell was

$$
d(\mathbf{S})=32.74-1.79 s_{x}+1.3 s_{y}+0.13 s_{z}
$$

and the positional gradient function,

$$
d(\mathbf{S})=32.74+2.22|\mathbf{S}| \cos \Theta_{\mathrm{GS}} \text {. }
$$

The coordinates of the orientation $\mathbf{G}$ of the gradient were

$$
g_{x}=-0.81, \quad g_{v}=0.59, \quad g_{z}=0.06 \text {. }
$$

The $F$ tests in the ANOVA and multiple regression were statistically significant ( $p<0.01$ for both analyses).

The 3-D positional gradient can be represented in 3 plots in which the discharge rate is plotted against position (distance) in

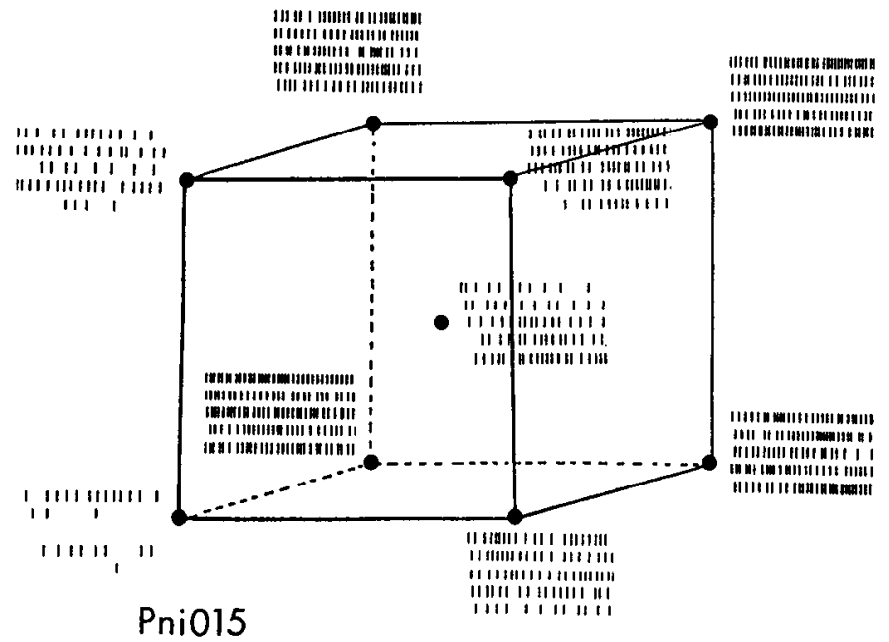

Figure 3. Impulse activity (5 trials) of a cell during holding of the hand at positions indicated. Each trace is $0.6 \mathrm{sec}$ long.

each of the 3 coordinate axes. This is illustrated in Figure 4. The plot for a particular axis was derived by setting the position for the other 2 axes equal to zero and solving Equation 6 for various values of the axis retained. When the positions for the other 2 axes are not zero but constant, and the position for the third axis varies, then lines parallel to those shown are produced, i.e., with the same slope but different intercepts.

The slopes of each of the 3 lines in Figure 4 equal the respective coefficients in Equation 6 . The SE of these coefficients were $0.125,0.090$, and 0.095 for the coefficients (see Eq. 6) $-1.79,1.3$, and 0.13 , respectively.

The visualization of the positional gradient as a variation of the intensity of discharge in 3-D space is more difficult, but an attempt was made to represent it in Figurc 5 (data from another cell). Each dot represents a position within the workspace; the intensity of the dot is proportional to the predicted cell discharge. The intensity of discharge increases gradually towards a certain part of the workspace.

The orientations of the positional gradients of all cells in which statistically significant functions were observed $(N=175)$ are shown in Figure 6 in the form of an equal-area projection plot. The procedure by which the plot is constructed was described by Schwartz et al. (1988). Briefly, the orientations of the gradients are considered as vectors of unit length with origin at the center of a unit sphere. The points where these vectors terminate are marked on the surface of the sphere. Finally, the surface of each of the 2 (upper and lower) hemispheres is projected onto a circular plane in such a way that areas on the hemisphere are projected to regions of equal area on the plane. The mathematical transformations are described in Watson (1983). In Figure 6, squares and crosses correspond to points in the upper and lower hemispheres, respectively. It can be seen that the orientations of the gradients are distributed throughout 3-D space.

Figure 7 shows the distributions of the parameters of the positional gradient functions obtained; in Figure $7, A, B$, the frequency distributions of the parameters $\int$ and $h$ (see Eq. 2) are shown. Figure $7 C$ is a scatter plot of $h$ versus $f$ in a $\log -\log$ scale; the 2 parameters were positively correlated $(r=0.823, p<$ $0.001, N=175$; log-log transformed data).

The choice of an origin for the positional gradient is arbitrary 

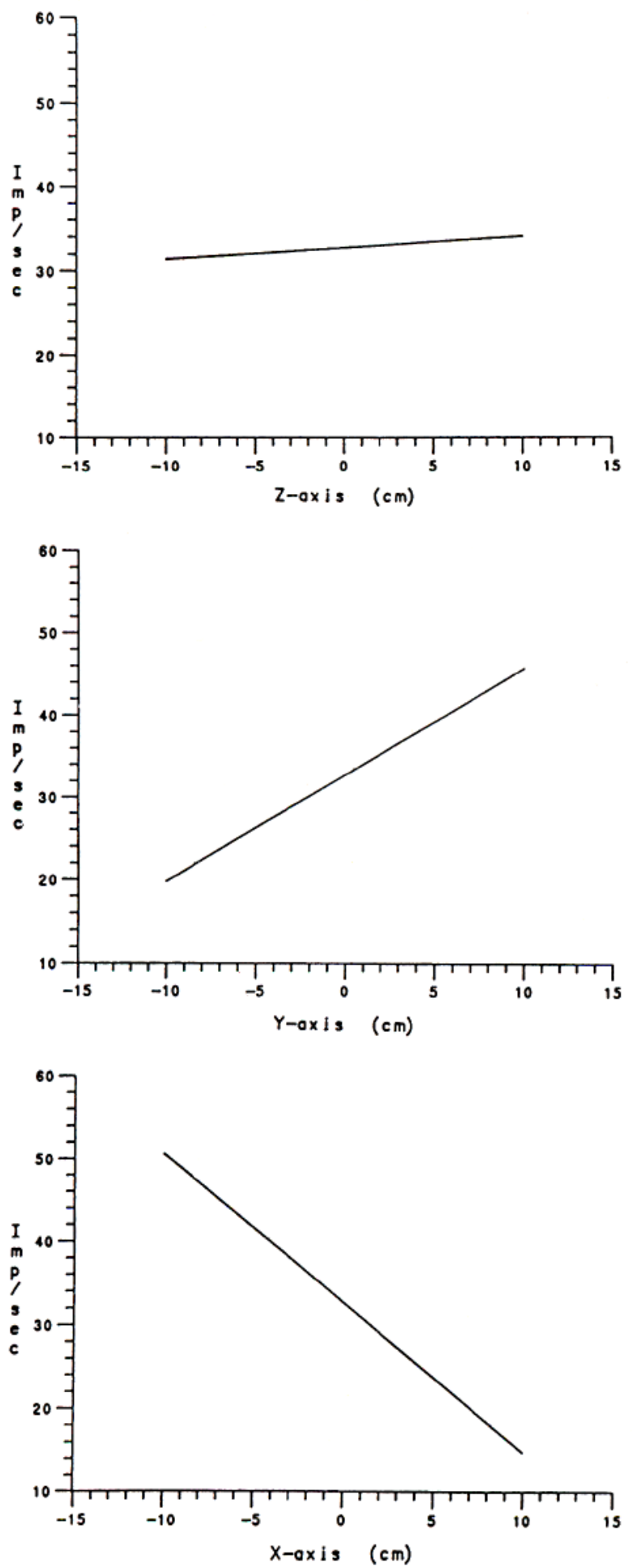

Figure 4. The 3-D positional gradient is illustrated as variation in the predicted discharge rate during holding at various positions (distances) along a coordinate axis ( $X, Y, Z$ axis, from bottom to top). Equation 6 was used to generate the plots.

and is assumed to be associated with a coordinate system that would move with the body of the animal. We have chosen the center button (see Fig. 1) as our coordinate origin for convenience in making computations and to allow comparisons with directed movements made in the center $\rightarrow$ out task in which all movements began at the center button. This was possible be-

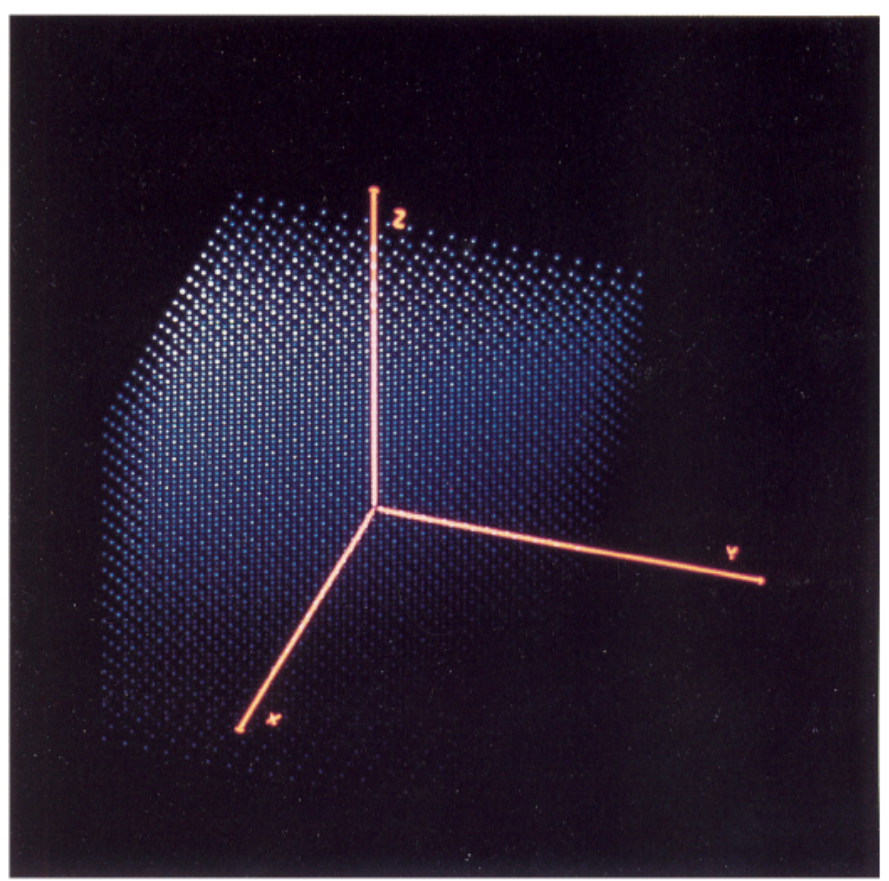

Figure 5. The 3-D positional gradient from another cell illustrated as a 3-D plot. Each dot represents a position within the workspace. The intensity of a dot is proportional to the intensity of cell discharge predicted by the positional gradient function.

cause the animal's head was fixed during the experiments and the body was loosely restrained so that the body coordinate system remained in approximate alignment with the coordinate system of the apparatus.

Comparison of changes in activity during movement predicted from the positional gradient with the observed ones. The positional gradient function allows the prediction of changes in cell activity when the hand moves from one point to another, changes predicted from the static positional gradient alone. To what extent can these predicted changes account for the observed ones? This problem was investigated for the cells that possessed a statistically significant positional gradient. Consider a movement from the center to a peripheral light. The change in cell activity predicted by the positional gradient function equals onehalf of the difference between the static-hold activity at these 2 positions. This value was compared with the actual change in activity observed during the movement of the hand from the center to the same peripheral light. First, we wanted to know how well these pairs of values were correlated for each cell that possessed a positional gradient. For that purpose, a productmoment correlation coefficient was calculated for each cell $(n$ $=8$ pairs). Figure 8 shows the distribution of the 175 correlations obtained. Of those, $72 / 175(41.1 \%)$ were statistically significant $(p<0.05, R>0.707, d f=8-2=6)$. For these 72 cells the following regression equation was obtained (values are means \pm SEM from 72 individual regression equations):

$$
A=9.067( \pm 4.12)+2.77( \pm 0.33) B
$$

where $A$ is the observed change in discharge rate during the movement time, and $B$ is the change in discharge rate predicted by the positional gradient function. Equation 9 indicates that the observed changes in cell activity are more than twice as large as those predicted by the positional gradient. Thus, dynamic 
Figure 6. Equal-area plot of positional gradient orientations $(N=175)$. Data points are for right hand. See text for explanation.

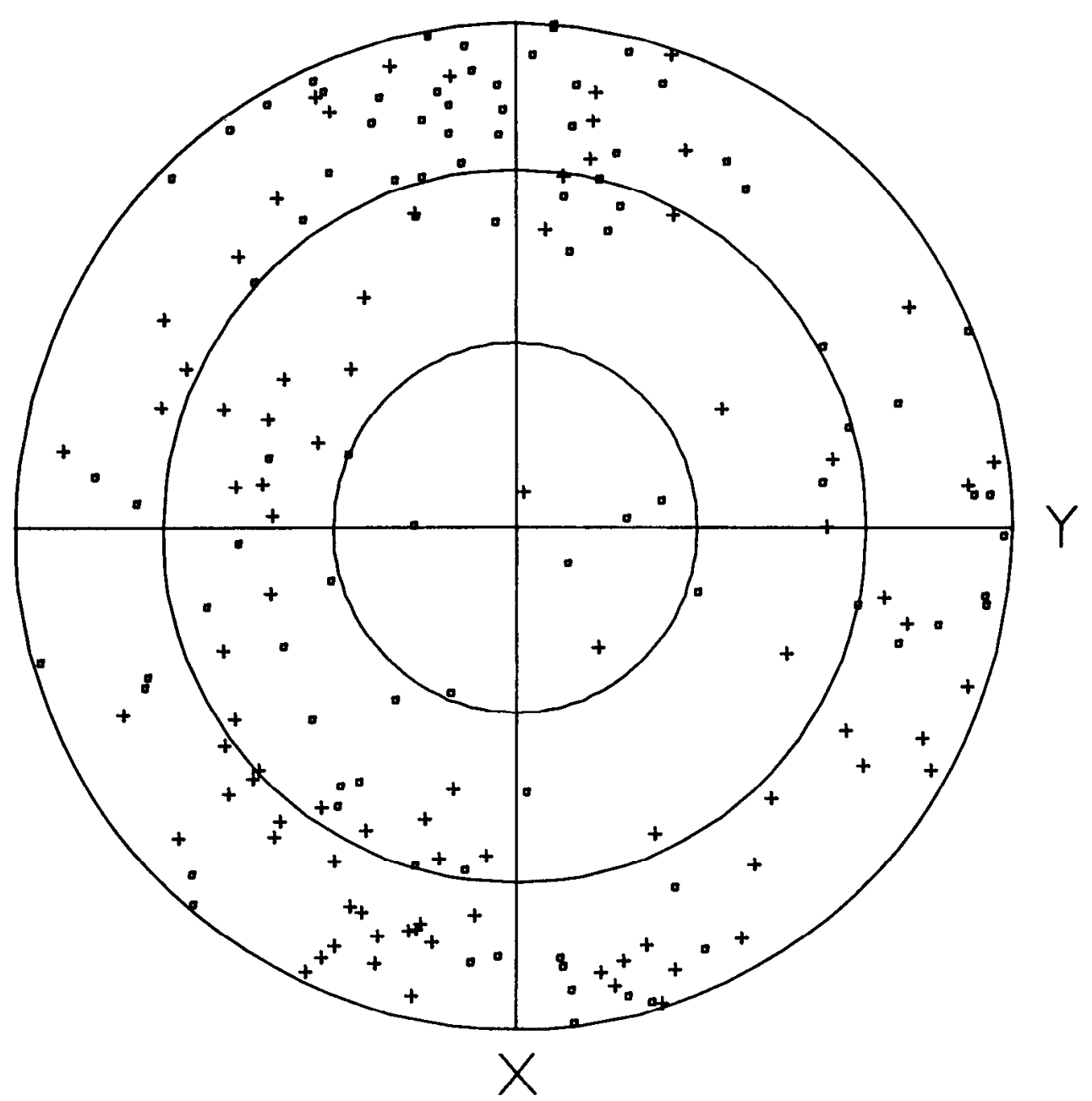

factors related to movement of the arm contribute substantially to the cell discharge (see also Georgopoulos and Massey, 1985).

Effects of difference in point of origin of the movement: parallel movements in the same direction. We examined neuronal and EMG activity when parallel movements were made in the same direction but at different regions in the workspace, during performance in the "parallel" task (Fig. 1C). Figure 9 shows the EMG activity of the acromiodeltoid muscle in this task. The plots are arranged as pairs corresponding to parallel movements made in the same direction, as indicated in the center diagram. It can be seen that the EMG activity differs substantially between equal-amplitude movements made in the same direction but in different parts of space.

Figure 10 illustrates an example from a neuron studied in this task; in this case the changes in cell activity were similar for movements in the same direction, although they differed for the pair of movements 4 and 7.

The overall relations between changes in discharge rates associated with sets of parallel movements in the same direction are shown in the scatter plot of Figure 11. Each point corresponds to changes in discharge rates during the TET epoch of parallel movements made in the same direction but in different parts of space. The data are from cells that showed a statistically significant ANOVA in the parallel task ( 245 cells $\times 4$ pairs of parallel movements per cell $=980$ points). It can be seen that, overall, the changes in discharge rates tended to be similar for parallel movements; the correlation coefficient was $R=0.760$ $(p<0.001, N=980)$.

A statistical comparison of the discharge rates associated with parallel movements in the same direction was carried out using the LSD method in the ANOVA (see Materials and Methods). Of the 980 pairs of changes in discharge rates, 310 pairs $(31.6 \%)$ differed significantly from each other, but the remainder, 670 pairs, $(68.4 \%)$ did not. A different question concerns the number of cells that showed statistically significant differences between pairs of parallel movements. The proportions obtained were as follows: In $67 / 245(27.3 \%)$ cells, no statistically significant differences were found between any of the 4 pairs of parallel movements; in $88 / 245(35.9 \%)$ cells, only 1 pair was significantly different; in $57 / 245(23.3 \%)$ cells, 2 pairs; in $25 / 245(10.2 \%)$ cells, 3 pairs; and in $8 / 245(3.3 \%)$ cells, 4 pairs. These results indicate that there is a continuum with respect to differences in cell activity in relation to pairs of parallel movements, although most cells $(155 / 245,63.3 \%)$ showed significant changes in only 1 , or none, of the 4 pairs of parallel movements.

Neuronal population analysis. We used the analysis described in the preceding paper (Georgopoulos et al., 1988) to calculate the neuronal population vector for movements with various origins in the center $\rightarrow$ out and parallel tasks. We found the following. First, the direction of the population vector was congruent with the direction of the movement. An example is shown in Figure 12 for a movement in the parallel task. The 16 pairs 

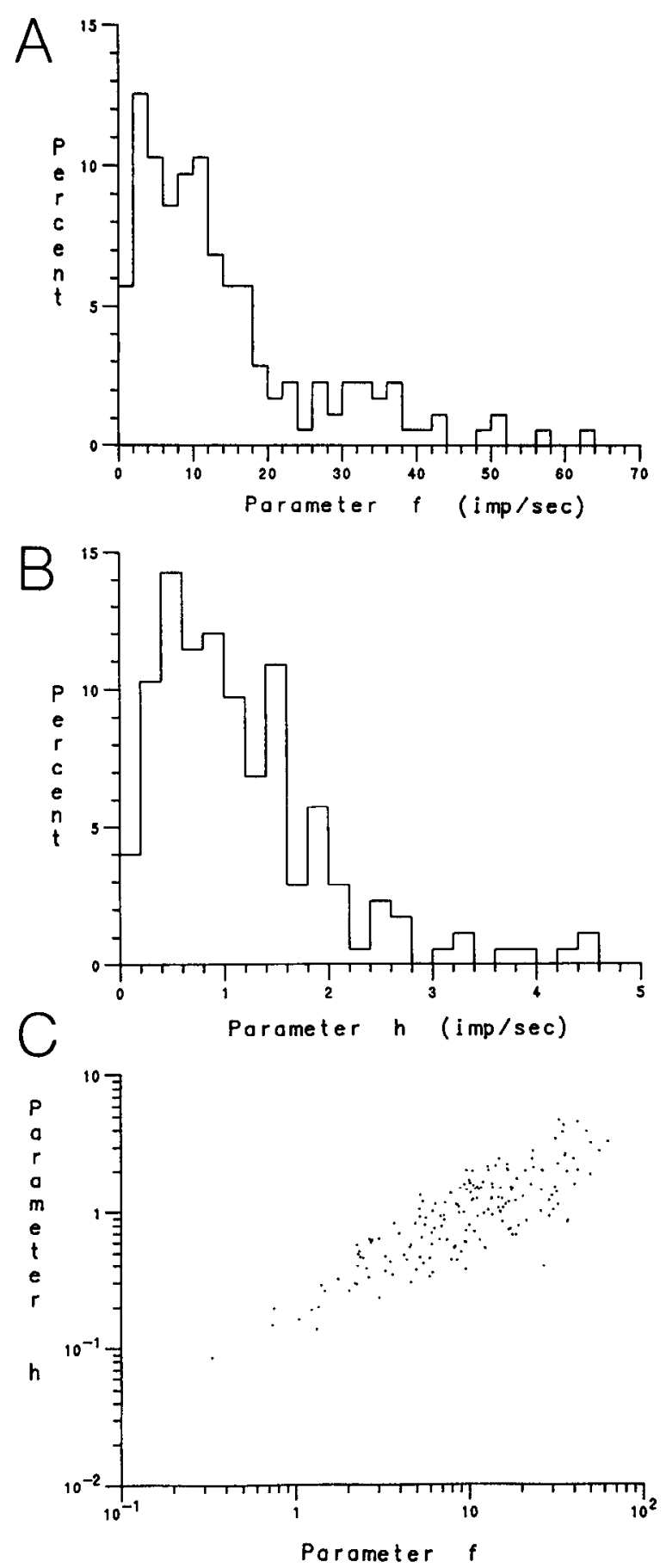

Figure 7. Distributions of parameters of positional gradient function $(N=175)$. $A$, Frequency distribution of parameter $f . B$, Frequency distribution of parameter $h$. $C$, Joint distribution of parameter $h$ versus parameter $f$.

of population and movement vectors were significantly correlated (spherical correlation of Fisher and Lee, 1986, $\rho=0.960$, $p<0.001$, randomized permutation method) with an average angle between the 2 vectors of $20.4^{\circ}$. The accuracy of prediction was almost identical in the 2 tasks: center $\rightarrow$ out task, $19.8^{\circ}$ (mean of 8 directions), $\rho=0.974, p<0.001$; parallel task, $20.9^{\circ}$ (mean of 8 directions), $\rho=0.840, p<0.001$ ). And second, the direction of the population vector calculated every $20 \mathrm{msec}$ during the reaction time predicted well the direction of the upcoming movement.

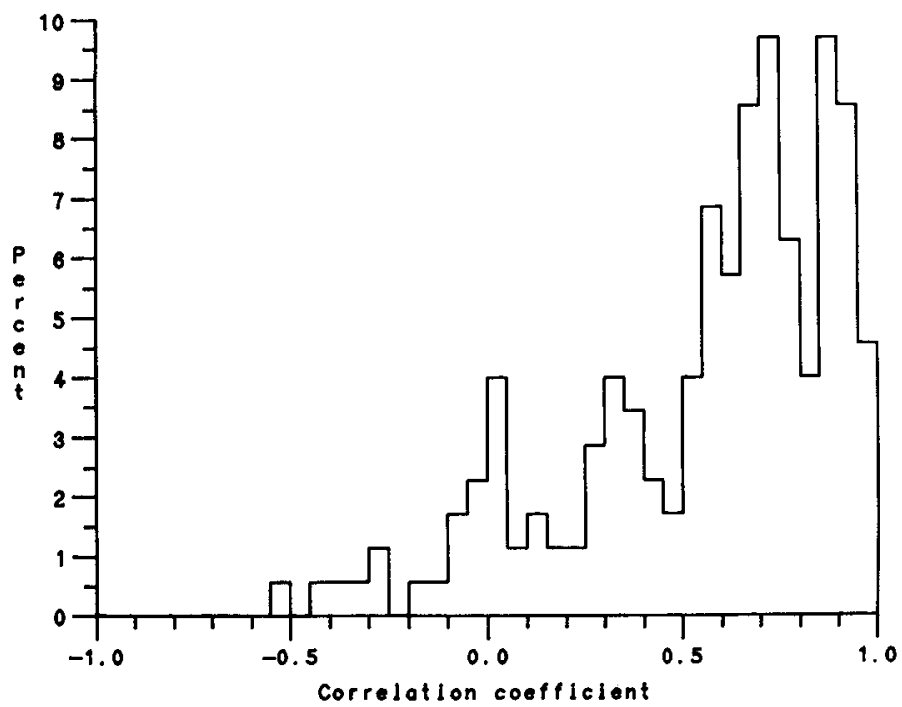

Figure 8. Distribution of correlation coefficients $(N=175)$ obtained between observed values of discharge rates during movement time and those predicted from the positional gradient function.

\section{Discussion}

The questions addressed in this study deal with the control by the motor cortex of free arm movements in 3-D space. There are 3 findings of this study. First, there are orderly relations between the activity of single cclls in the motor cortex and the position at which the arm is actively maintained in space. Second, the changes in single-cell activity are similar for equalamplitude movements made in the same direction within different parts of the workspace, although a significant variation in cell activity exists. And third, the neuronal population vector predicts well the direction of movement for movements whose point of origin varies in space. These findings together with those described in the preceding papers (Georgopoulos et al., 1988; Schwartz et al., 1988) point to the arm area of the motor cortex as a key structure for the specification of the spatial aspects of reaching.

\section{Positional gradients}

Motor cortical cells frequently show initial increases or decreases in activity in relation to the initiation of a motor act, and then their activity stabilizes at a stcady-state level (Porter and Lewis, 1975; Cheney and Fetz, 1980; Georgopoulos et al., 1982; Murphy et al., 1985). In isometric tasks, this level has been shown to be a linear function of the magnitude of force exerted by the animal, for motor cortical cells that presumably project monosynaptically to motoneuronal pools innervating distal muscles (Cheney and Fetz, 1980). When the direction of the load was varied in 2 dimensions, the steady-state level of cell activity in the motor cortex varied sinusoidally with the direction of the load (Kalaska and Hyde, 1985). A different problem concerns the relations of this static motor cortical activity to the posture of the arm. An orderly, linear relation was found in previous studies between the static activity of cells in the motor cortex and the actively maintained position of the hand in 2-D space (Georgopoulos et al., 1984b), so that the frequency of cell discharge associated with various positions of the hand on a working surface could be described as a planar gradient with a particular orientation within the working surface, and a specific 


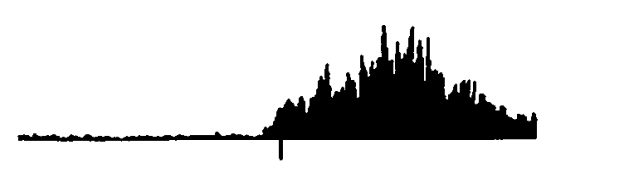

1
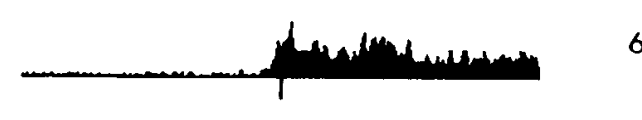

6

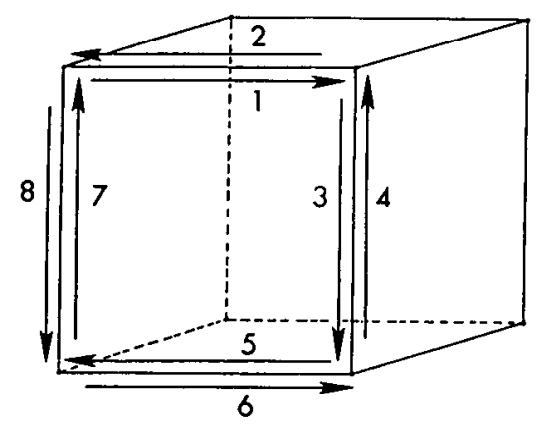

2

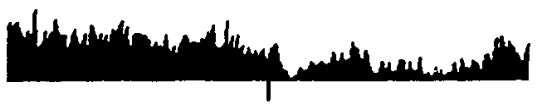

3

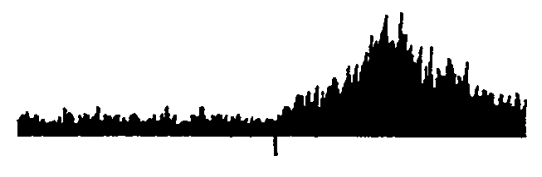

4
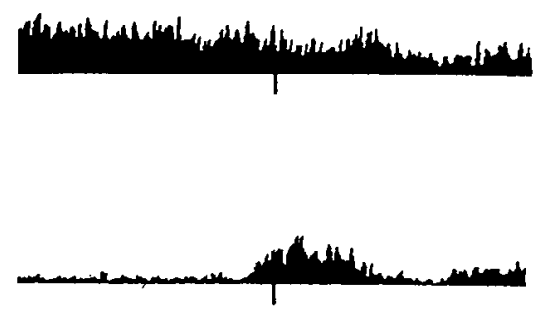

8

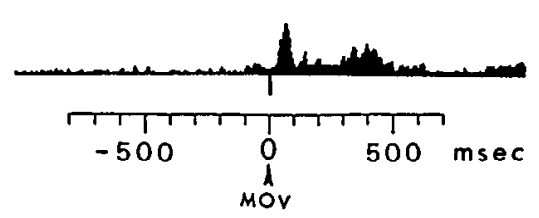

Figure 9. EMG activity of acromiodeltoid muscle in the parallel task. Each histogram is an average of 8 trials and is aligned to the onset of movement (MOV). Arrows indicate the direction of movement. Plots have been arranged in pairs corresponding to parallel movements, as identified by the numbers.

angle of elevation from it. Although the EMG activity of several muscles acting on the shoulder and the sagittal and coronal components of the shoulder angle also varied in a linear fashion with the position of the hand, the orientations of the 2-D positional gradients of the cells were not clustered around those dictated by the muscles or the joints but were distributed throughout 2-D space. This suggested that single cells in the motor cortex might relate to combinations of forces exerted by groups of muscles, or to combinations of angles at the elbow and/or shoulder joints, rather than reflecting a one-to-one relation (Georgopoulos et al., 1984b). The results of the present studies extend those observations to $3-\mathrm{D}$ space. Thus, the position of the hand in 3-D space and the posture of the arm associated with this position are represented in a linear fashion by the static-hold discharge rate in many motor cortical cells, and the orientations of the neuronal positional gradients are distributed throughout 3-D space. It is more difficult to visualize this relation in 3-D space but an attempt was made to illustrate it in Figure 5. Another way to describe it follows from Equation 1: If the position changes only along 1 of the 3 axes, then the frequency of discharge will be a linear function of position in that axis; thus, the coefficients $f_{x}, f_{y}, f_{z}$ in Equation 1 represent the slopes of these linear functions in each of the 3 axes (Fig. 4). In fact, this approach was used as the experimental design in the study of the relations between the frequency of discharge of "fixation" neurons in area 7 of the posterior parietal cortex and the position of the gaze in 3-D space (Sakata et al., 1980). In that study, monkeys were trained to fixate a light whose position was varied systematically along each of the $X, Y, Z$ axes in 3-D space. It was found that in many cells the frequency of cell discharge varied in an orderly fashion with the position of the light along a particular axis.

\section{Coding of the direction of movement in space for movements of various origins}

An important question regarding the role of motor cortex in controlling arm movements in space concerns the effects on single-cell activity, and possible coding schemes, of the point of origin of the movement in space. The results described in the preceding 2 papers (Georgopoulos et al., 1988; Schwartz et al., 1988) were based on movements that started from the same point at the center of the workspace. In the present experiments, we studied cell activity both in the center $\rightarrow$ out task ( 8 movement directions) and when 4 pairs of parallel movements were made in the same direction from various points of origin in different parts of the workspace ( 8 additional directions).

An obvious effect of the point of origin of the movement was the different static discharge rates associated with holding the hand at different positions in spacc, as discussed in the preceding section. This led us to the hypothesis that it is the change in 

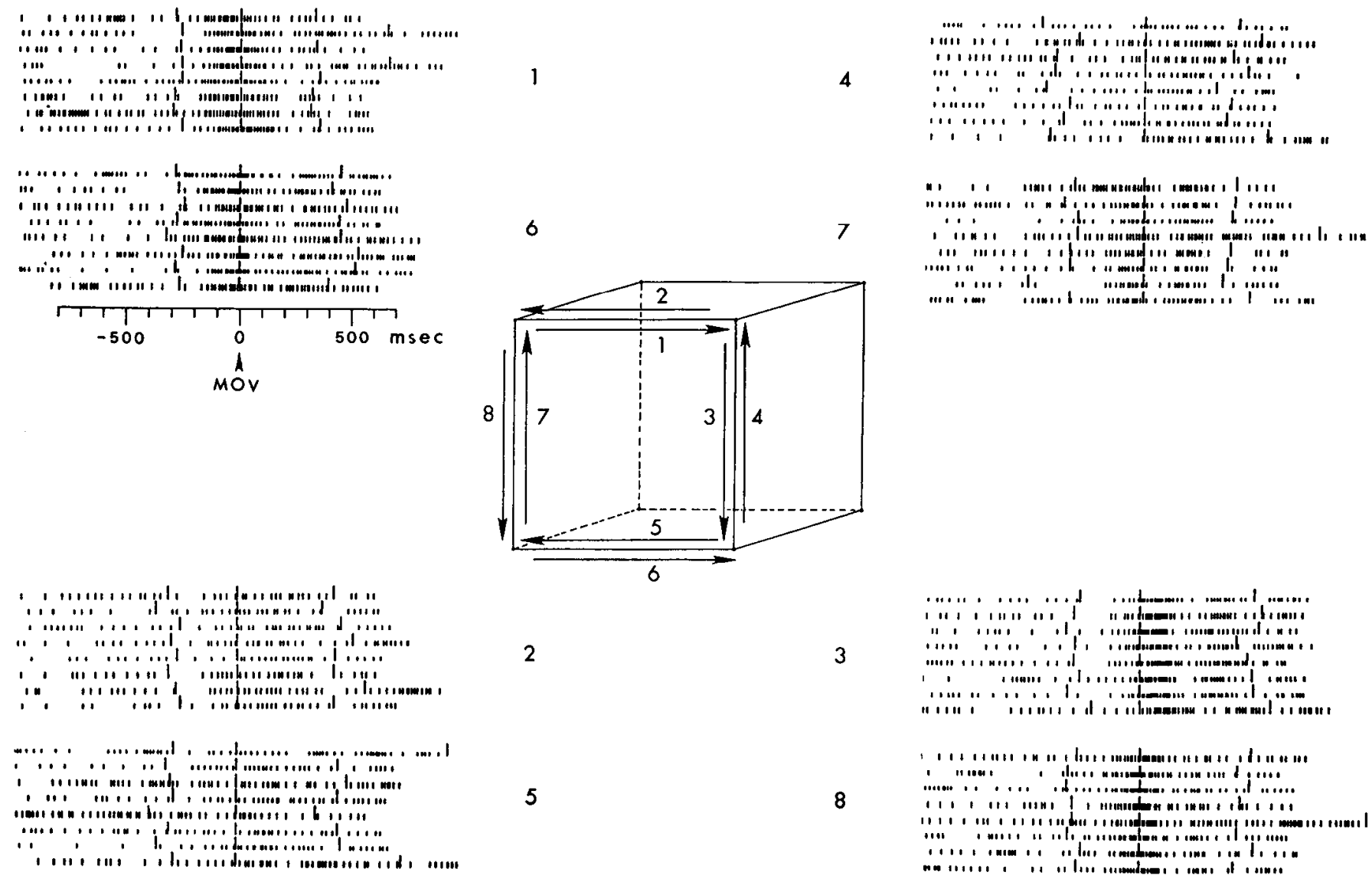

2

3

5

8

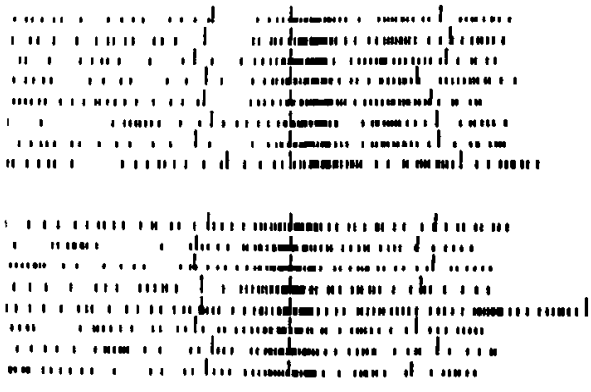

Pio092

Figure 10. Impulse activity of a cell in the parallel task. Five repetitions are shown aligned to the onset of movement (MOV). Longer vertical bars preceding the movement onset indicate the time of target onset. Same arrangement as in Figure 9.

cell activity that may relate to movement, whereas the total cell activity may relate to a combination of both static and dynamic factors. Thus, we compared the changes in cell activity from the ongoing static-hold discharge rate for parallel movements made in the same direction but within different parts of the workspace. The results at the single-cell level showed that the changes in activity associated with movements in the same direction were similar for many pairs of parallel movements $(68.4 \%)$, that is, independent of the spatial location of the trajectory, although for the remaining $31.6 \%$ of parallel movement pairs these changes differed. However, it is remarkable that in $27.3 \%$ of the cells, no significant differences were found in the discharge between any of the 4 parallel movements, and in an additional $35.9 \%$ significant changes were found in only 1 of the 4 pairs. In contrast to neuronal activity, the EMG activity from the arm was quite different, as the hand moved from different origins in parallel directions (Fig. 9).

\section{Population coding of the direction of movements from different origins}

The results of the analysis of the changes in single-cell activity during parallel movements indicate that there is a continuum in these changes and that movements in particular directions do not engage exclusive subsets of the population. However, when these cells were considered as an ensemble, the population analysis showed that the neuronal population vector provided unique information about the direction of the movement. In fact, the degree of congruence between the population vector and the direction of movement was almost identical (ca. $20^{\circ}$ ) for the case of movements made from the same or from different points of origin. These results reinforce the main theme of the population approach; namely, that specificity and uniqueness resides with the neuronal ensemble rather than its individual elements.

\section{The arm area of the motor cortex as a processor of spatial- motor information}

The results of this study and those described in the preceding papers (Georgopoulos et al., 1988; Schwartz et al., 1988; see also Georgopoulos et al., 1984a; Crutcher et al., 1985) reveal a picture of the arm area of the motor cortex as a processor of spatial-motor information. This view may be more applicable to the arm area of the motor cortex, than to its hand and finger area, because of differences required in the control of reaching versus the control of manipulatory movements.

There is littlc doubt that the arm area of the motor cortex participates in the control of proximal arm movements, as has been documented by electrical stimulation (Woolsey et al., 1950; Kwan et al., 1978; Sessle and Wiesendanger, 1982), lesion (Passingham et al., 1983), and single-cell recording studies in behaving monkeys (Porter and Lewis, 1975; Murphy et al., 1982, 1985; Georgopoulos et al., 1982, 1986; Schwartz et al., 1988). Movements at the shoulder and elbow joints are usually coordinated into reaching movements in immediate extrapersonal 
Figure 11. Scatter plot of changes in cell activity (from the control period) during parallel movements made in the same direction but at different parts of space. Movements in a pair were randomly assigned to the ordinate or the abscissa $(N=245$ cells $\times 4$ pairs of parallel movements per cell $=980$ points).

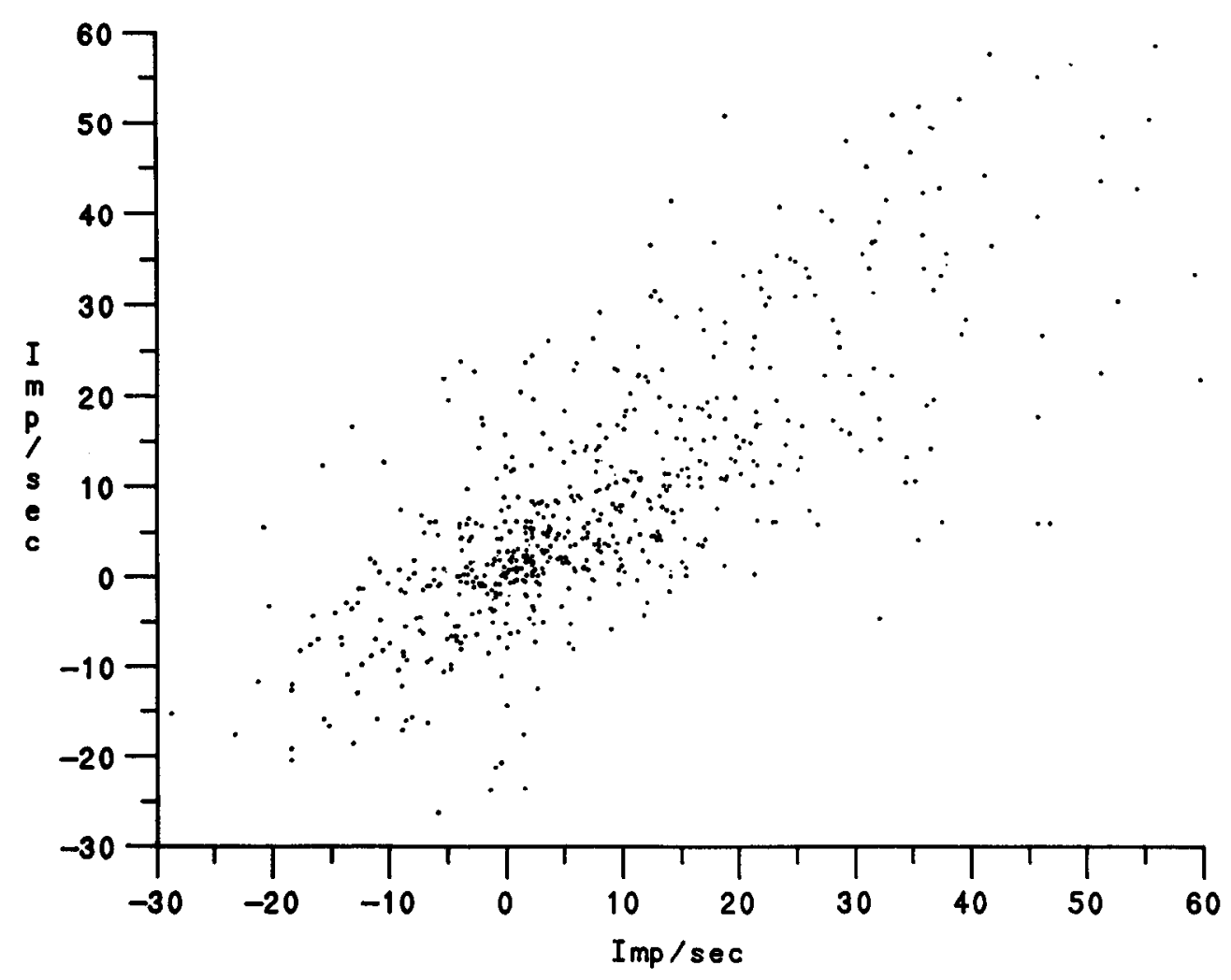

Thc present and previous studies, mentioned above, have identified 3 major factors -2 spatial and 1 temporal - that relate to the neuronal activity in the arm area of the motor cortex. The spatial factors are the direction of movement and the position of the arm in space, and the temporal factor is the duration for which the target of the movement stays on before it changes to another location in tasks in which the animal is trained to move towards a target light (Georgopoulos et al., 1983b). Surprisingly, the relations of the activity of single cells to the amplitude of the movement are neither as frequent nor as strong as those to direction (Schwartz and Georgopoulos, 1987; see also Hamada and Kubota, 1979), but a signal related to the instantaneous velocity of the upcoming movement may be present in the population discharge (Georgopoulos et al., 1988).

In retrospect, it may not be surprising that spatial attributes of the reaching movement provide good descriptors of the motor cortical activity in the arm area, for a major part of the organization and implementation of these movements seems to reside in highly and intricately organized spinal and other subcortical circuits. The studies of Lundberg and collaborators during the past 12 years on the function and behavioral significance for reaching of the C3-C4 propriospinal system (see Lundberg, 1979, for a review, and Alstermark et al., 1981, for behavioral studies) have provided an insight into the spinal control of reaching and pointed to new ways by which the motor cortical control of these circuits might be exerted, ways that are different from the commonly postulated "upper motor neuron," one-toone control of muscles involved in reaching. The results of the studies on the C3-C4 propriospinal system were discussed in the first paper of this series (Schwartz et al., 1988) and will not be repeated here. Suffice it to say that the motor cortical signals engaging that system, or other spinal interneuronal systems, necd not be coded in muscle coordinates: those spinal circuits could indeed effect hard-wired transformations between spa-
Figure 12. Neuronal population vector (P) for a movement $(\mathbf{M})$ in the parallel task. 
tially coded motor cortical signals and the ultimate activation of multiple motoneuronal pools subserving reaching. The appropriate connectivity between motor cortical output and spinal circuits could be finalized in the early months of life, during which the corticospinal system is still developing and the accuracy of reaching improves.

\section{References}

Alstermark, B., A. Lundberg, U. Norrsell, and E. Sybirska (1981) Integration in descending motor pathways controlling the forelimb in the cat. 9. Differential behavioral defects after spinal cord lesions interrupting defined pathways from higher centres to motoneurones. Exp. Brain Res. 42: 299-318.

Cheney, P. D., and E. E. Fetz (1980) Functional classes of primate corticomotoneuronal cells and their relation to active force. J. Neurophysiol. 44: 773-791.

Crutcher, M. D., A. B. Schwartz, and A. P. Georgopoulos (1985) Representation of movement direction in primate motor cortex in the absence of immediate movement. Soc. Neurosci. Abstr. 11: 1273.

Fisher, N. I., and A. J. Lee (1986) Correlation coefficients for random variables on a unit sphere or hypersphere. Biometrika 73: 159-164.

Georgopoulos, A. P., and J. T. Massey (1985) Static versus dynamic effects in motor cortex and area 5: Comparison during movement time. Behav. Brain Res. 18: 159-166.

Georgopoulos, A. P., J. F. Kalaska, R. Caminiti, and J. T. Massey (1982) On the relations between the direction of two-dimensional arm movements and cell discharge in primate motor cortex. J. Neurosci. 2: $1527-1537$.

Georgopoulos, A. P., R. Caminiti, J. F. Kalaska, and J. T. Massey (1983a) Spatial coding of movement: A hypothesis concerning the coding of movement direction by motor cortical populations. Exp. Brain Res. (Suppl.) 7: 327-336.

Georgopoulos, A. P., J. F. Kalaska, R. Caminiti, and J. T. Massey (1983b) Interruption of motor cortical discharge subserving aimed arm movements. Exp. Brain Res. 49: 327-340.

Georgopoulos, A. P., J. F. Kalaska, M. D. Crutcher, R. Caminiti, and $J$. T. Massey (1984a) The representation of movement direction in the motor cortex: Single cell and population studies. In Dynamic Aspects of Neocortical Function, G. M. Edelman, W. E. Gall, and W. M. Cowan, eds., pp. 501-524, Wiley, New York.

Georgopoulos, A. P., R. Caminiti, and J. F. Kalaska (1984b) Static spatial effects in motor cortex and area 5: Quantitative relations in a two-dimensional space. Exp. Brain Res. 54: 446-454.

Georgopoulos, A. P., A. B. Schwartz, and R. E. Kettner (1986) Neuronal population coding of movement direction. Science 233: 14161419.

Georgopoulos, A. P., R. E. Kettner, and A. B. Schwartz (1988) Primate motor cortex and free arm movements to visual targets in threedimensional space. II. Coding of the direction of movement by a neuronal population. J. Neurosci. 8: 2928-2937.
Hamada, I., and K. Kubota (1979) Monkey pyramidal tract neurons and changes of movement parameters in visual tracking. Brain Res. Bull. 4: 249-257.

Ingelfinger, J. A., F. Mosteller, L. A. Thibodeau, and J. H. Ware (1983) Biostatistics in Clinical Medicine, Macmillan, New York.

Kalaska, J. F., and M. L. Hyde (1985) Area 4 and area 5: Differences betwecn the load-dependent discharge variability of cells during active postural fixation. Exp. Brain Res. 59: 197-202.

Kettner, R. E., A. B. Schwartz, and A. P. Georgopoulos (1986) Population coding of 3-dimensional movement direction in primate motor cortex: Effects of changes in origin and direction of movement. Soc. Neurosci. Abstr. 12: 257.

Kwan, H. C., W. A. MacKay, J. T. Murphy, and Y. C. Wong (1978) Spatial organization of precentral cortex in awake primates. II. Motor outputs. J. Neurophysiol. 41:1120-1131.

Lundberg, A. (1979) Integration in a propriospinal motor centre controlling the forelimb in the cat. In Integration in the Nervous System, H. Asanuma and V. S. Wilson, eds., pp. 47-69, Igaku-Shoin, Tokyo.

Murphy, J. T., H. C. Kwan, W. A. MacKay, and Y. C. Wong (1982) Precentral unit activity correlated with angular components of a compound arm movement. Brain Res. 246: 141-145.

Murphy, J. T., Y. C. Wong, and H. C. Kwan (1985) Sequential activation of neurons in primate motor cortex during unrestrained forelimb movement. J. Neurophysiol. 53: 435-445.

Passingham, R. E., V. H. Perry, and F. Wilkinson (1983) The longterm effects of removal of sensorimotor cortex in infant and adult rhesus monkeys. Barin 106: 675-705.

Porter, R., and M. McLewis (1975) Relationship of neuronal discharges in the precentral gyrus of monkeys to the performance of arm movements. Brain Res. 98: 21-36.

Sakata, H., H. Shibutani, and K. Kawano (1980) Spatial properties of visual fixation neurons in posterior parietal association cortex of the monkey. J. Neurophysiol. 43: 1654-1672.

Schwartz, A. B., and A. P. Georgopoulos (1987) Relations between the amplitude of 2-dimensional arm movements and single cell discharge in primate motor cortex. Soc. Neurosci. Abstr. 13: 244.

Schwartz, A. B., R. E. Kettner, and A. P. Georgopoulos (1988) Primate motor cortex and free arm movements to visual targets in threedimensional space. I. Relations between single cell discharge and direction of movement. J. Neurosci. 8: 2913-2927.

Sessle, B. J., and M. Wiesendanger (1982) Structural and functional definition of the motor cortex in the monkey (Macaca fascicularis). J. Physiol. (Lond.) 323: 245-265.

Snedecor, G. W., and W. G. Cochran (1980) Statistical Methods, 7th ed., Iowa State University Press, Ames, Iowa.

Watson, G. S. (1983) Statistics on Spheres, Wiley, New York.

Woolsey, C. N., P. H. Settlage, D. R. Meyer, W. Spencer, P. Hamuy, and A. M. Travis (1950) Patterns of localization in the precentral and "supplementary" motor areas and their relation to the concept of a premotor area. Res. Publ. Assoc. Res. Nerv. Ment. Dis. 30: 238264. 